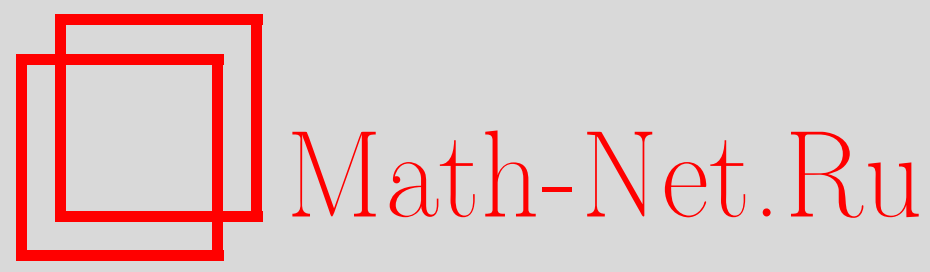

А. В. Жибер, С. Я. Старцев, Интегралы, решения и существование преобразований Лапласа линейной гиперболической системы уравнений, Матем. заметки, 2003, том 74, выпуск 6, 848-857

DOI: https://doi.org/10.4213/mzm322

Использование Общероссийского математического портала Math-Net.Ru подразумевает, что вы прочитали и согласны с пользовательским соглашением http://www . mathnet.ru/rus/agreement

Параметры загрузки:

IP : 3.95 .254 .165

26 апреля 2023 г., 15:54:01

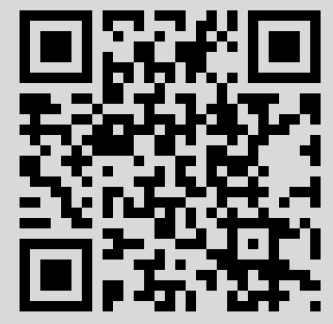




\title{
ИНТЕГРАЛЫ, РЕШЕНИЯ И СУЩЕСТВОВАНИЕ ПРЕОБРАЗОВАНИЙ ЛАПЛАСА ЛИНЕЙНОЙ ГИПЕРБОЛИЧЕСКОЙ СИСТЕМЫ УРАВНЕНИЙ
}

\author{
А. В. Жибер, С. Я. Старцев
}

Преобразования и инварианты Лапласа обобщаются на случай гиперболической системы уравнений, и исследуются условия их существования. Доказано, что система уравнений допускает преобразование Лапласа тогда и только тогда, когда найдется матрица ранга $k$, где $k$ - дефект соответствующего инварианта Лапласа, переводящая в решение этой системы любой вектор, составленный из функций от одной из независимых переменных. Показано, что для существования завершающейся нулем цепочки инвариантов Лапласа необходимым является наличие полного набора интегралов у исходной системы уравнений и полного набора решений, зависящих от произвольных функций, у сопряженной системы. Предъявлен пример, показывающий, что эти условия не являются достаточными для существования преобразования Лапласа.

Библиографоя: 13 названий.

1. Введение. Хорошо известно (см., например, [1], [2]), что любое скалярное уравнение вида

$$
L(u) \equiv u_{x y}+a(x, y) u_{x}+b(x, y) u_{y}+c(x, y) u=0,
$$

с отличньм от нуля инвариантом Лапласа $h=a_{x}+a b-c$ допускает дифференциальную подстановку $v=u_{y}+a u$, называемую преобразованием Лапласа и переводящую решения уравнения (1) в решения уравнения

$$
L_{1}(v) \equiv v_{x y}+\left(a-\frac{h_{y}}{h}\right) v_{x}+b(x, y) v_{y}+\left[\left(a-\frac{h_{y}}{h}\right) b+b_{y}-h\right] v=0 .
$$

Условие $h \neq 0$ гарантирует нам “обратимость” этого преобразования: дифференциальная подстановка $u=\left(v_{x}+b v\right) / h$ переводит решения уравнения (2) обратно в решения (1).

Вышеописанные преобразования лежат в основе так называемого каскадного метода интегрирования Лапласа: проделав преобразования Лапласа несколько раз, мы можем, в некоторых "хороших" случаях, прийти к уравнению вида

$$
\left(\frac{\partial}{\partial x}+b\right)\left(\frac{\partial}{\partial y}+\hat{a}\right) w=0
$$

Работа выполнена при финансовой поддержке Российского фонда фундаментальных исследований, грант № 02-01-00144. 
которое легко интегрируется, а затем, воспользовавшись обратными преобразованиями Лапласа, получить из решений этого уравнения решения исходного уравнения (1). Тот факт, что уравнение (1) может быть записано в виде

$$
\left(\frac{\partial}{\partial x}+b\right)\left(\frac{\partial}{\partial y}+a\right) u-h u=0
$$

гарантирует нам, что цепочка преобразований Лапласа не оборвется раньше, чем мы приведем уравнение к желаемому виду.

Наблюдающийся в последнее десятилетие всплеск интереса к преобразованиям и инвариантам Лапласа обусловлен тем, что в ряде недавних работ (см. [3]-[10]) были обнаружены тесные связи между некоторыми важными свойствами скалярного нелинейного уравнения

$$
u_{x y}=F\left(x, y, u, u_{x}, u_{y}\right),
$$

такими, как точная интегрируемость, наличие симметрий и дифференциальных подстановок, и свойствами цепочки инвариантов Лапласа линеаризации

$$
Z_{x y}-F_{u_{x}} Z_{x}-F_{u_{y}} Z_{y}-F_{u} Z=0
$$

этого уравнения. В дополнение к этому в работах [5], [11], [12] было продемонстрировано, что преобразования Лапласа могут быть распространены и на некоторые уравнения, имеющие вид, отличньй от (4).

Следующим естественным шагом в подобных исследованиях представляется обобщение вьшеупомянутых результатов на системы уравнений вида (4). Основой для такого обобщения должны являться преобразования Лапласа для линейных систем уравнений вида (1), подобно тому, как результаты, известные для скалярного уравнения (4), основываются на преобразованиях Лапласа для скалярного уравнения (1). Между тем, хотя инварианты и преобразования Лапласа для скалярных линейных уравнений известны уже более сотни лет, преобразования Лапласа для систем линейных уравнений, по-видимому, никем еще не изучались: отдельные попытки подобного исследования были предприняты лишь совсем недавно в работах [10], [13].

Как будет показано в следующем пункте, основная трудность, возникающая при обобщении преобразований и инвариантов Лапласа на случай систем (как линейных, так и нелинейных), состоит в том, что не любая система вида (1) допускает преобразование Лапласа. Исследованию различных условий существования преобразований Лапласа и посвящена настоящая работа.

В п. 3 мы в предположении существования цепочки преобразований Лапласа, завершающейся нулевьп инвариантом Лапласа, строим явные формулы для нахождения интегралов и решений системы (1) и, тем самым, показываем, что необходимым условием существования такой цепочки является наличие полного набора интегралов у исходной системы уравнений и полного набора решений, зависящих от произвольных функций, у сопряженной к (1) системы. В п. 4 получен критерий существования преобразования Лапласа в терминах решений системы (1) - таким критерием является наличие матрицы, переводящей в решение системы любой вектор функций, зависящих от одной из независимых переменных, и имеющей ранг, совпадающий с дефектом соответствующего инварианта Лапласа. В п. 5 приводится пример системы уравнений, обладающей по 
каждой из характеристик полньми наборами интегралов и зависящих от произвольных функций решений, но не допускаюшей преобразование Лапласа - тем самым показано, что полученньй в п. 4 критерий не может быть усилен за счет включения в решение производных от произвольной функции, а необходимые условия, приведенные в п. 3, не являются достаточными для существования преобразования Лапласа.

2. Преобразования Лапласа систем уравнений. Нетрудно проверить, что в скалярном случае верна формула

$$
\left(\frac{\partial}{\partial y}+a_{1}\right) L=L_{1}\left(\frac{\partial}{\partial y}+a\right)
$$

где $a_{1}=a-h_{y} / h$, а дифференциальные операторы $L$ и $L_{1}$ заданы формулами $(1)$ и $(2)$ соответственно. Эта формула означает, что ядро $L$ переводится оператором $\partial / \partial y+a$ в ядро $L_{1}$, и является ключевой при изучении преобразований Лапласа для скалярных уравнений.

Рассмотрим теперь системы линейных уравнений (1): будем в дальнейшем $u$ считать $n$-мерньм вектором, а коэффициенты $a, b$ и $c$-квадратными матрицами. Взяв за основу для определения инварианта Лапласа формулу (3) и попытавшись подобрать матрицу $a_{1}$ и оператор $L_{1}$ так, чтобы выполнялась формула (5), получим

$$
h=a_{x}+b a-c, \quad L_{1}=\left(\frac{\partial}{\partial y}+a_{1}\right)\left(\frac{\partial}{\partial x}+b\right)-h, \quad h_{y}-h a+a_{1} h=0 .
$$

Переписав $L_{1}$ в виде $(\partial / \partial x+b)\left(\partial / \partial y+a_{1}\right)-h_{1}$, получим формулу для инварианта Лапласа $h_{1}$ преобразованного уравнения

$$
h_{1}=\frac{\partial a_{1}}{\partial x}+\left[b, a_{1}\right]-\frac{\partial b}{\partial y}+h .
$$

Многократно повторяя вьшеприведенное рассуждение, получаем следующее

ОПРЕДЕЛЕНИЕ 1 . Матрицы $h_{i}, i \geqslant 0$, определяемые рекуррентными формулами

$$
\begin{gathered}
\frac{\partial h_{i}}{\partial y}-h_{i} a_{i}+a_{i+1} h_{i}=0 \\
h_{i+1}=\frac{\partial a_{i+1}}{\partial x}+\left[b, a_{i+1}\right]-\frac{\partial b}{\partial y}+h_{i}
\end{gathered}
$$

и первыми членами

$$
a_{0}=a, \quad h_{0}=a_{x}+b a-c,
$$

называются $у$-инвариантами Лапласа системы уравнений (1).

Результатом $i$-кратного применения $y$-преобразования Лапласа к системе (1) будем называть систему уравнений

$$
L_{i}(u) \equiv\left(\frac{\partial}{\partial x}+b\right)\left(\frac{\partial}{\partial y}+a_{i}\right) u-h_{i} u=0 .
$$


При таком выборе $a_{i}, h_{i}$ и $L_{i}$ дифференциальные подстановки $v=u_{y}+a_{i} u$, называемые преобразованиями Лапласа, будут переводить любое решение системы уравнений $L_{i}(u)=0$ в решение системы $L_{i+1}(v)=0$.

Здесь, видимо, необходимо пояснить, что преобразования Лапласа переводят решения $L_{i}(u)=0$ в решения $L_{i+1}(v)=0$ в силу того, что $a_{i}, h_{i}$ и $L_{i}$ в вьшеприведенном определении выбраны так, чтобы вьполнялись соотношения

$$
\left(\frac{\partial}{\partial y}+a_{i+1}\right) L_{i}=L_{i+1}\left(\frac{\partial}{\partial y}+a_{i}\right) .
$$

Аналогичным образом (поменяв местами $x$ и $y$, а также $a$ и $b$ в вышеприведенном определении) мы можем определить $x$-инварианты и $x$-преобразования Лапласа. В дальнейшем мы будем приводить результаты, касающиеся лишь одной из характеристик системы (1), - результаты, относящиеся к другой характеристике, получаются из них с помощью вьшеуказанной замены.

Нетрудно видеть, что в случае вырожденности матрицы $h_{i}$, определяющее соотношение (6), вообще говоря, неразрешимо относительно $a_{i+1}$ и мы не можем гарантировать существования инвариантов Лапласа начиная с того момента, как очередной инвариант стал вырожденным. Между тем, именно такая ситуация и представляет наибольший интерес, так как преобразования Лапласа обьчно делаются с целью достичь уравнения с инвариантом Лапласа, равным нулю, для чего, как показывает анализ примеров, приходится проходить через ряд вырожденных, но не равных нулю инвариантов. Отсутствиегарантии существования преобразований Лапласа является основной трудностью при исследовании систем уравнений, так как многие известные в скалярном случае утверждения об инвариантах Лапласа достаточно легко переносятся на случай систем (см. [13]), если эту трудность проигнорировать и насильственно предположить существование инвариантов.

3. Построение интегралов и решений. Изучим сначала необходимые условия существования цепочки преобразований Лапласа: предположим, что мы можем проделать преобразование Лапласа системы (1) несколько раз, и выясним, какими свойствами должна обладать система уравнений, допускающая такие преобразования. Поскольку в скалярном случае преобразования Лапласа делаются, как правило, с целью получить уравнение с нулевым инвариантом Лапласа, то мы, по большому счету, ничего не теряем, предположив дополнительно, что на каком-то шаге инвариант Лапласа обратится в нуль.

Нетрудно увидеть, что система уравнений, удовлетворяющая вьшеприведенным предположениям, характеризуется наличием у нее полного набора так назьваемых $y$-интегралов ( $x$-интегралов), т.е. функций, зависящих от $x, y, u$ и производных $u$ по $y$ (по $x$ ), которые при подстановке в них в качестве $u$ любого решения уравнения (1) дают функцию, не зависящую от $x$ (от $y$ ). Говоря более строго, верно следующее

УТВЕРЖДЕНИЕ 1. Пусть система уравнений (1) допускает т-кратное у-преобразование Лапласа и ее т-й у-инвариант равен нулю. Тогда у этой системы имеется набор $W$ из п функиионально независимых $у$-интегралов, задаваемый формулой

$$
W=g(x, y)\left(\frac{\partial}{\partial y}+a_{m}\right) \ldots\left(\frac{\partial}{\partial y}+a\right) u,
$$


где $a_{i}$ определяются по формуле (6), а в качестве g взята невырожденная матрища, удовлетворяющая соотношению $\partial g / \partial x-g b=0$.

ДокАЗАТЕльСТво. Многократно применив формулу (7), получим

$$
L_{m}\left(\frac{\partial}{\partial y}+a_{m-1}\right) \ldots\left(\frac{\partial}{\partial y}+a\right)=\left(\frac{\partial}{\partial y}+a_{m}\right) \ldots\left(\frac{\partial}{\partial y}+a_{1}\right) L
$$

Учитьвая, что $L_{m}=(\partial / \partial x+b)\left(\partial / \partial y+a_{m}\right)$ и $g(\partial / \partial x+b)=\partial / \partial x \circ g$, мы можем умножить обе части этой формулы на $g$ и переписать ее в виде

$$
\frac{\partial}{\partial x}\left[g(x, y)\left(\frac{\partial}{\partial y}+a_{m}\right) \ldots\left(\frac{\partial}{\partial y}+a\right) u\right]=g(x, y)\left(\frac{\partial}{\partial y}+a_{m}\right) \ldots\left(\frac{\partial}{\partial y}+a_{1}\right) L(u)
$$

Из последней формулы видно, что ее левая часть равна нулю всегда, когда $u$ является решением системы (1).

Заметим, что интегралы являются объектом, важньм при исследовании скорее нелинейных систем уравнений. Для линейных же систем более правильным применением преобразований Лапласа представляется построение решений. Однако для описанной во введении схемы построения решений необходимьм является наличиепреобразования, обратного к преобразованию Лапласа, в то время как в случае систем уравнений такого обратного преобразования не имеется. Тем не менее, мы можем обойти это затруднение, строя системы уравнений, переводящиеся в систему (1) прямьм преобразованием Лапласа.

Для построения такой цепочки "прообразов" системы (1) удобно рассмотреть формально сопряженную к ней систему уравнений $L^{\top}(u)=0$. Напомним, что сопряжение операторов есть линейная унарная операция, на множестве обычных линейных операторов (матриц) совпадающая с транспонированием, а на множестве дифференциальных операторов определяемая правилами

$$
\left(\frac{\partial}{\partial x}\right)^{\top}=-\frac{\partial}{\partial x}, \quad\left(\frac{\partial}{\partial y}\right)^{\top}=-\frac{\partial}{\partial y} \quad \text { и } \quad(P \circ R)^{\top}=R^{\top} \circ P^{\top}
$$

для любой пары операторов $P$ и $R$, где $S^{\top}$ обозначает оператор, сопряженный к оператору $S$. В частности, сопряженная к (1) система имеет вид

$$
L^{\top}(u) \equiv u_{x y}-a^{\top} u_{x}-b^{\top} u_{y}+\left(c^{\top}-a_{x}^{\top}-b_{y}^{\top}\right) u=0
$$

Если эта система допускает $y$-преобразование Лапласа, то верна формула

$$
\left(\frac{\partial}{\partial y}+\hat{a}_{1}\right) L^{\top}=\left(L^{\top}\right)_{1}\left(\frac{\partial}{\partial y}-a^{\top}\right)
$$

формальное сопряжение которой дает нам

$$
\left(\frac{\partial}{\partial y}+a\right)\left(\left(L^{\top}\right)_{1}\right)^{\top}=L\left(\frac{\partial}{\partial y}-\hat{a}_{1}^{\top}\right)
$$


Таким образом, решения системы (1) получаются преобразованием Лапласа $u=v_{y}-$ $\hat{a}_{1}^{\top} v$ из решений системы $\left(\left(L^{\top}\right)_{1}\right)^{\top}(v)=0$.

Если же нам удалось проделать преобразование Лапласа $m$ раз и $m$-й $y$-инвариант Лапласа системы (8) обратился в нуль, то получаем уравнение

$$
\left(\left(L^{\top}\right)_{m}\right)^{\top}(w) \equiv\left(\frac{\partial}{\partial y}-\hat{a}_{m}^{\top}\right)\left(\frac{\partial}{\partial x}+b\right) w=0
$$

которое легко интегрируется и $m$-кратным преобразованием Лапласа

$$
u=\left(\frac{\partial}{\partial y}-\hat{a}_{1}^{\top}\right) \cdots\left(\frac{\partial}{\partial y}-\hat{a}_{m}^{\top}\right) w
$$

переводится в систему (1). Взяв в качестве решения системы (9) вектор $g(x, y) f(y)$, где $f(y)$ - произвольный $n$-мерньй вектор, а матрица $g$ удовлетворяет соотношению $\partial g / \partial x+b g=0$, получаем следующее

УТВЕРЖДЕНИЕ 2. Пусть система уравнений $L^{\top}(u)=0$, формально сопряжсенная к системе (1), допускает т-кратное у-преобразование Лапласа и ее т-й у-инвариант равен нулю. Тогда

$$
u=\left(\frac{\partial}{\partial y}-\hat{a}_{1}^{\top}\right) \cdots\left(\frac{\partial}{\partial y}-\hat{a}_{m}^{\top}\right)(g(x, y) f(y)),
$$

где через $\hat{a}_{i}$ обозначены коэффициенты при $\partial / \partial x$ в $\left(L^{\top}\right)_{i}$, а невырожденная матрииа $g$ удовлетворяет соотношению $\partial g / \partial x+b g=0$ иявляется решением системы (1) для любого п-мерного вектора $f(y)$.

Утверждения 1 и 2 означают, что необходимыми условиями существования у системы (1) обращающейся в нуль цепочки инвариантов Лапласа являются наличие полного набора интегралов у системы (1) и полного набора решений, зависящих от произвольных функций, у сопряженной к ней системы (8). Поясним, что в настоящей статье мы считаем набор интегралов (решений) полным, если строки (столбцы) коэффициентов при старших производных $u$ в интегралах (при старших производных произвольной функции в решениях) образуют невырожденную матрицу размерности $n$. (В контексте данной статьи такого определения вполне достаточно, поскольку в ней мы оперируем лишь интегралами и решениями, линейно зависящими от старших производных.)

В следующем пункте статьи мы покажем, что для существования преобразования Лапласа необходимо наличие зависяшего от произвольных функций решения и у самой исходной системы (1).

4. Критерий существования преобразования Лапласа. Из работы [10] известно следующее

УТВЕРЖДЕНИЕ 3 (Соколов). Соотношение (6) разрешимо относительно $a_{i+1}$ тогда и только тогда, когда дифференциальный оператор $\partial / \partial y+a_{i}$ переводит ядро $h_{i}$ снова в ядро $h_{i}$.

Для полноты изложения приведем доказательство этого утверждения. 
ДокАЗАТЕЛЬСТво. Прямьм вычислением нетрудно убедиться, что (6) равносильно операторному соотношению

$$
\left(\frac{\partial}{\partial y}+a_{i+1}\right) \circ h_{i}=h_{i} \circ\left(\frac{\partial}{\partial y}+a_{i}\right)
$$

из которого вытекает необходимость сохранения ядра $h_{i}$ при действии на него оператором $\partial / \partial y+a_{i}$.

Пусть теперь известно, что $\left(\partial / \partial y+a_{i}\right)\left(\operatorname{ker} h_{i}\right) \subset \operatorname{ker} h_{i}$. Обозначим через $z$ матрицу, столбцы которой образуют базис ядра $h_{i}$. Тогда $h_{i}\left(z_{y}+a_{i} z\right)=0$. Учитывая, что $h_{i} z=0$ и, следовательно, $h_{i} z_{y}=-\left(h_{i}\right)_{y} z$, мы получаем $\left(\left(h_{i}\right)_{y}-h_{i} a_{i}\right) z=0$. В силу теоремы $\Phi$ редгольма последнее соотношение означает разрешимость (6) относительно $a_{i+1}$. Утверждение 3 доказано.

Опираясь на это утверждение, мы можем доказать следующий критерий существования преобразования Лапласа.

ТЕорема 1. Система уравнений (1) допускает у-преобразование Лапласа тогда и только тогда, когда найдется матрица $S(x, y)$ такая, что $u=S(x, y) f(x)$ является решением системы (1) для любого п-мерного вектора $f(x)$ и сумма рангов матрии, $S$ и р равна $n$, где $n$ - число зависимых переменных системы (1), $a$ через $h$ обозначен нулевой $y$-инвариант Лапласа әтой системы $\left(h=a_{x}+b a-c\right)$.

ДокАЗАТЕльСтво. Нетрудно проверить, что наличие решения указанного вида равносильно вьполнению следующих соотношений:

$$
\left(\frac{\partial}{\partial y}+a\right) S=0, \quad h S=0
$$

Из последнего равенства видно, что мы можем выбрать базис ядра $h$ из столбцов матрицы $S$ и любой элемент из ker $h$ представить в виде $S z$, где $z$-некоторый вектор. Учитывая первое из соотношений (10), мы видим, что оператор $\partial / \partial y+a$ переводит вектор $S z$ в вектор $S z_{y}$, которьй тоже лежит в ядре $h$. Это с учетом утверждения 3 доказывает достаточность наличия вьшеуказанного решения для сушествования преобразования Лапласа.

Прежде чем перейти к доказательству необходимости, заметим, что наличие у системы (1) преобразования Лапласа является свойством, инвариантным относительно замен переменных вида $u=T(x, y) \hat{u}$ - подставив формулы $h=T \hat{h} T^{-1}$ и $a=T \hat{a} T^{-1}-$ $T_{y} T^{-1}$ в соотношение (6) (при $i=0$ ), мы убеждаемся, что разрешимость этого соотношения сохранится при такой замене.

Выберем теперь невырожденную матрицу $G(x, y)$ так, что $\partial G / \partial y+a G=0$. Заменой переменных $u=G \hat{u}$ перейдем от системы (1) к системе

$$
\left(\frac{\partial}{\partial x}+\hat{b}\right) \frac{\partial \hat{u}}{\partial y}-\hat{h} \hat{u}=0, \quad \hat{h}=G^{-1} h G .
$$

Пусть ранг $\hat{h}$ равен $m$. Перенумеровав компоненты вектора $\hat{u}$ соответствующей заменой переменных, мы без нарушения общности можем считать, что первые $m$ столбцов в 
матрице $\hat{h}$ линейно независимы. Так как остальные столбцы могут быть представлены как линейная комбинация первых $m$ столбцов, найдется матрица

$$
T=\left(\begin{array}{cc}
E_{m} & Z \\
0 & E_{n-m}
\end{array}\right)
$$

где $E_{i}$ - единичные матрицы размера $i$ такие, что у матрищы $\hat{h} T$ все столбцы, начиная с $(m+1)$-го, нулевые. Сделав замену переменных $\hat{u}=T \tilde{u}$, придем к системе уравнений

$$
\left(\frac{\partial}{\partial x}+\tilde{b}\right)\left(\frac{\partial}{\partial y}+T^{-1} T_{y}\right) \tilde{u}-\tilde{h} \tilde{u}=0, \quad \tilde{h}=T^{-1} \hat{h} T .
$$

В качестве базиса ядра $\tilde{h}$ мы можем выбрать векторы $e_{m+1}, \ldots, e_{n}$, где $e_{i}-$ вектор, у которого $i$-я компонента равна 1 , а остальные компоненты равны нулю. Заметив, что

$$
T^{-1}=\left(\begin{array}{cc}
E_{m} & -Z \\
0 & E_{n-m}
\end{array}\right), \quad T^{-1} T_{y}=\left(\begin{array}{cc}
0 & Z_{y} \\
0 & 0
\end{array}\right),
$$

мы видим, что $\partial / \partial y+T^{-1} T_{y}$ может переводить в ядро $\tilde{h}$ все вектора $e_{i}, i=\overline{m+1, n}$, лишь при условии $Z_{y}=0$. Тогда матрица

$$
S=G\left(\begin{array}{c}
Z \\
E_{n-m}
\end{array}\right)
$$

удовлетворяет соотношениям (10), что и требовалось доказать.

5. Недостаточность наличия интегралов и решений высших порядков для существования преобразования Лапласа. Приведенный в теореме 1 критерий имеет тот недостаток, что дает гарантию возможности сделать лишь однократное преобразование Лапласа. В приложениях же, как правило, необходимо делать несколько последовательных преобразований Лапласа. С другой стороны, в скалярном случае преобразования и инварианты Лапласа применяются в основном для выявления уравнений, обладающих интегралами и (или) решениями вида $\sum_{i=0}^{m} \alpha_{i}(x, y) f^{(i)}$, где $f-$ произвольная функция от одной из независимых переменных, и естественным является стремление обосновать применимость преобразований Лапласа к системам уравнений с аналогичньми свойствами, вьведя существование цепочки преобразований Лапласа необходимой длины из наличия у системы (1) полных наборов интегралов и решений вышеуказанного вида. Теорема 1 и утверждения 1 и 2, казалось бы, свидетельствуют в пользу такой связи между интегралами, решениями и существованием преобразований Лапласа. В действительности, однако, такая связь отсутствует, что показывает следующий

ПРИмЕР. Рассмотрим систему уравнений

$$
\begin{gathered}
{\left[\frac{\partial^{2}}{\partial x \partial y}-\frac{3}{x+y}\left(\begin{array}{ll}
1 & 1 \\
0 & 0
\end{array}\right) \frac{\partial}{\partial x}-\left(\begin{array}{cc}
\frac{1}{x+y} & \frac{1}{x+y}-y \\
0 & -x
\end{array}\right) \frac{\partial}{\partial y}\right.} \\
\left.+\left(\begin{array}{cc}
\frac{4}{(x+y)^{2}} & \frac{4}{(x+y)^{2}}-2 \\
0 & 0
\end{array}\right)\right]\left(\begin{array}{l}
u \\
v
\end{array}\right)=0 .
\end{gathered}
$$


Эта система обладает двумя функционально независимыми $x$-интегралами

$$
\begin{aligned}
& w_{1}=\frac{u_{x}+u_{x}}{(x+y)^{3}}-\frac{u+v}{(x+y)^{4}}+\frac{v}{(x+y)^{2}}, \\
& w_{2}=v_{x}+x v
\end{aligned}
$$

и двумя функционально независимьми $y$-интегралами

$$
\begin{aligned}
\bar{w}_{1}= & -\frac{u_{y y y}}{x+y}+3 \frac{u_{y y}+v_{y y}}{(x+y)^{2}}-6 \frac{u_{y}+v_{y}}{(x+y)^{3}}+6 \frac{u+v}{(x+y)^{4}} \\
& -v_{y y y} e^{x^{2} / 2} \int\left(\frac{y e^{-x^{2} / 2}}{x+y}-\frac{e^{-x^{2} / 2}}{(x+y)^{2}}\right) d x, \\
\bar{w}_{2}= & e^{x^{2} / 2} v_{y y y} .
\end{aligned}
$$

Кроме того, система (11) обладает полньм набором решений

$$
\begin{aligned}
\left(\begin{array}{l}
u \\
v
\end{array}\right)= & {\left[\left(\begin{array}{cc}
(x+y)^{3} & -1 \\
0 & 1
\end{array}\right) \frac{\partial}{\partial x}-\left(\begin{array}{cc}
2(x+y)^{2}+2 & x+y \\
-2 & 0
\end{array}\right)\right]\left(\begin{array}{l}
g_{1}(x) \\
g_{2}(x)
\end{array}\right) } \\
& +\left(\begin{array}{cc}
x+y & (x+y) \int\left(\frac{1}{(x+y)^{2}}-\frac{y}{x+y}\right) e^{-x^{2} / 2} d x \\
0 & e^{-x^{2} / 2}
\end{array}\right)\left(\begin{array}{l}
f_{1}(y) \\
f_{2}(y)
\end{array}\right)
\end{aligned}
$$

при любом выборе функций $f_{1}(y), f_{2}(y), g_{1}(x), g_{2}(x)$.

Утверждение 2 наводит нас на мысль, что, возможно, достаточным условием существования преобразований Лапласа является наличие полных наборов интегралов и зависящих от произвольных функций решений у сопряженной системы. Приведенньй выше пример удовлетворяет и этому предположению: сопряженная к (11) система обладает как интегралами, так и решениями необходимого вида. Это следует из того общего факта, что система (1) допускает набор интегралов вида

$$
\sum_{i=0}^{k} \alpha_{i}(x, y) \frac{\partial^{i} u}{\partial y^{i}}
$$

тогда и только тогда, когда сопряженная к (1) система обладает решениями вида

$$
\sum_{i=0}^{k-1} \beta_{i}(x, y) f^{(i)}(y),
$$

где $\alpha_{i}, \beta_{i}$ - некоторые матрищы, причем $\beta_{k-1}=\alpha_{k}^{\top}$, a $f(y)$-произвольный вектор функций (см. доказательство утверждения 3 в работе [13, с. 150]).

Таким образом, как система (11), так и сопряженная к ней система обладают по каждой из характеристик как полным набором интегралов, так и полным набором решений, зависяших от произвольных функций - вьполнены все возможные условия, которые, гипотетически, должны были бы гарантировать наличие преобразования Лапласа. Однако, выписав для (11) соотношение (6), мы легко убеждаемся в том, что это соотношение неразрешимо уже при $i=0$ и система (11) не допускает $y$-преобразования Лапласа.

Заметим, что имеется целое семейство уравнений, обладающих аналогичными свойствами, но для простоты изложения мы выбрали из этого семейства один конкретньй представитель.

Авторы благодарят В. В. Соколова и С. П. Царева за обсуждение результатов этой работы. 


\section{СПИСОК ЦИТИРОВАННОЙ ЛИТЕРАТУРЫ}

[1] Darboux G. Leçons sur la théorie générale des surfaces et les applications geometriques du calcul infinitesimal. V. 1-4. Paris: Gauthier-Villars, 1896.

[2] Трикоми $\Phi$. Лекции по уравнениям в частных производных. М.: ИЛ, 1957.

[3] Жиибер А. В., Соколов В. В., Старцев С. Я. О нелинейньх гиперболических уравнениях, интегрируемых по Дарбу // Докл. РАН. 1995. Т. 343. №6. С. 746-748.

[4] Sokolov V. V., Zhiber A. V. On the Darboux integrable hyperbolic equations // Phys. Lett. A. 1995. V. 208. P. 303-308.

[5] Anderson I. M., Kamran N. The variational bicomplex for second order scalar partial differential equations in the plane // Duke Math. J. 1997. V. 87. № 2. P. 265-319.

[6] Anderson I. M., Juras M. Generalized Laplace invariant and the method of Darboux // Duke Math. J. 1997. V. 89. № 2. P. 351-375.

[7] Царев С. П. Факторизация линейных дифференциальных операторов с частными производными и метод Дарбу интегрирования нелинейных уравнений с частными производными // ТМФ. 2000. Т. 122. № 1. С. 144-160.

[8] Старцев С. Я. О дифференциальных подстановках типа преобразования Миуры // ТМФ. 1998. T. 116. № 3. С. 336-348.

[9] Старцев С. Я. О гиперболических уравнениях, допускающих дифференциальные подстановки // ТМФ. 2001. Т. 127. №1. С. 63-74.

[10] ЖКибер А. В., Соколов В. В. Точно интегрируемые уравнения лиувиллевского типа // УМH. 2001. Т. 56. №1. С. 63-106.

[11] Ферапонтов Е. В. Преобразования Лапласа систем гидродинамического типа в инвариантах Римана // ТМФ. 1997. Т. 110. № 1. С. 86-98.

[12] Адлер В. Э., Старцев С. Я. О дискретных аналогах уравнения Лиувилля // ТМФ. 1999. T. 121. № 2. C. 271-284.

[13] Старцев С. Я. Об инвариантах Лапласа систем гиперболических уравнений // Комплексный анализ, дифференциальные уравнения, численные методы и приложения. Т. 3. Уфа: Институт математики с ВЦ РАН, 1996. С. 144-154.

Институт математики с ВЦ Уфимского научного центра РАН

Поступило

E-mail :

01.08 .2002

zhiber@imat.rb.ru

starts@imat .rb.ru 